\title{
L'aménagement intégré d'un grand fleuve : le Rhône A. Conception générale et effets sur les crues et les nappes phréatiques
}

\author{
Integrated development of a large river: the Rhône \\ A. General design and effect on floods \\ and subsurface water layers
}

\author{
P. Savey \\ Directeur des Etudes et Travaux \\ à la Compagnie Nationale du Rhône
}

\section{La Compagnie Nationale du Rhône}

L'aménagement du Rhône sur les $500 \mathrm{~km}$ de son cours français, fut entrepris à grande échelle, à la fin de la seconde guerre mondiale, par la Compagnie Nationale du Rhône.

Il est aujourd'hui en voie d'achèvement, après les efforts de toute une génération d'ingénieurs et de techniciens.

Sur les 19 chutes prévues, 16 sont en exploitation, une en construction, et les deux dernières en cours d'enquête publique (Fig. 1).

Il s'agit d'un aménagement à buts multiples destiné principalement :

- à produire de l'énergie (16,9 TWh ont été livrés au réseau en 1981),

- à assurer la navigation à grand gabarit sur $300 \mathrm{~km}$ entre Lyon et la mer,

- et à contribuer au développement de l'agriculture riveraine (54000 ha remembrés, 34000 ha irrigués, 41000 ha protégés contre les crues en 1981).

La Compagnie Nationale du Rhône, à la fois Maitre d'Oeuvre et Maître d'Ouvrage, a assuré la totalité de l'ingénierie générale de ces aménagements. En outre, elle en assure l'exploitation en collaboration avec EDF.

La conception générale des aménagements

\section{Le Rhône}

Le Rhône, parvenant à la mer, a un débit d'étiage de $580 \mathrm{~m}^{3} / \mathrm{s}$ et un débit semi-permanent de $1460 \mathrm{~m}^{3} / \mathrm{s}$. Il transporte en année moyenne, 50 milliards de $\mathrm{m}^{3}$ d'eau. Sa plus grande crue connue (1856) est de $12000 \mathrm{~m}^{3} / \mathrm{s}$, et la crue, millénaire est estimée à $14000 \mathrm{~m}^{3} / \mathrm{s}$ (Fig. 2).

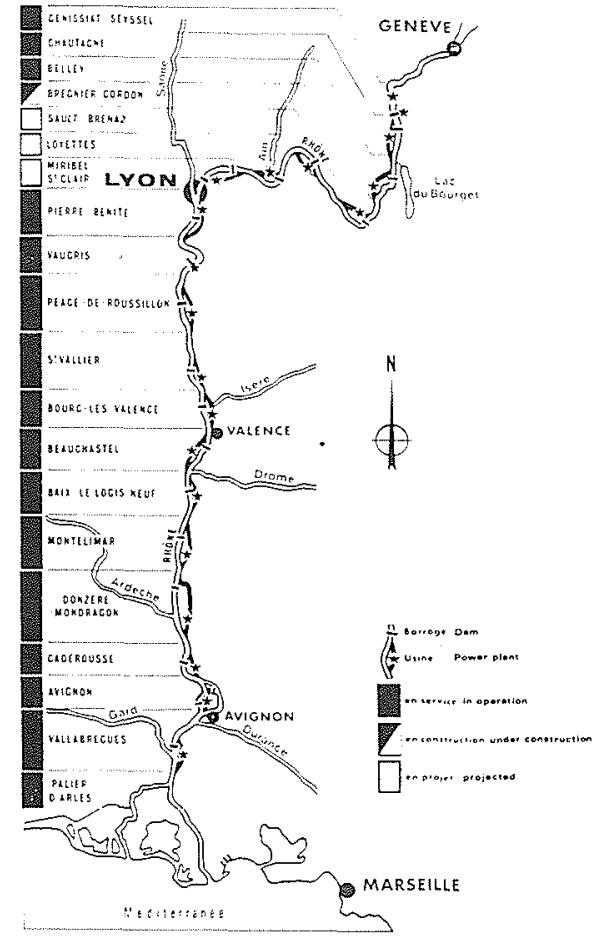

Figure 1 - Plan général de l'aménagement du Rhône.

La pente est variable suivant les secteurs

- entre $3 \%$ dans la partie amont du Haut-Rhône,

- $1 \%$ dans la région de Montélimar

- et seulement $0,01 \%$ lorsqu'il parvient à la mer.

Pour tirer le parti maximal d'un tel fleuve, il faudrait pouvoir réaliser en tête un important réservoir de quelques dizaines de milliards de $\mathrm{m}^{3}$ permettant d'accroître les étiages et d'écrêter les crues. Mais l'occupation humaine importante de ses rives (près de 2 mil- 


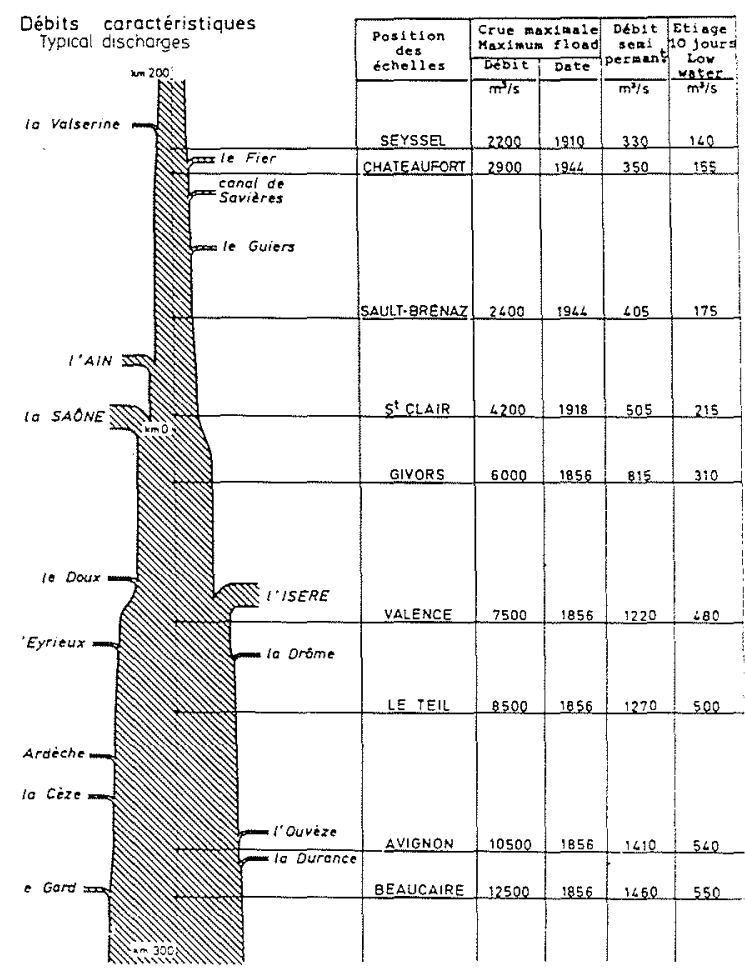

Figure 2 - Hydrologie du Rhône.

lions d'habitants au total) et la richesse agricole de sa vallée interdisent la réalisation d'une telle retenue.

\section{Un projet qui économise l'espace}

De ce fait, la contrainte dominante de l'aménagement du Rhône est la recherche de l'économie des emprises. C'est elle qui a conduit à diviser l'équipement en chutes de hauteur faible ou moyenne (de 6 à $21 \mathrm{~m}$ avec une exception de $70 \mathrm{~m}$ ). En outre, pour chacune de ces chutes, la C.N.R. a réduit l'importance des retenues, d'une part à l'aide d'endiguements latéraux, d'autre part en réalisant des canaux de dérivation. Ainsi, on a pu épargner complètement le domaine bâti et très largement le domaine agricole. Au total, les emprises sont de l'ordre de $8 \%$ de celles qu'aurait impliqué un projet hydroélectrique classique avec grand barrage et vaste retenue (Fig. 3 ).

Cette faiblesse des retenues (Fig. 4) en regard du débit du fleuve (la plupart d'entre elles sont remplies en quelques heures par le débit moyen), entraîne à son tour, deux conséquences importantes.

La première est qu'en aucun cas les retenues ne prennent un caractère lacustre, il ne se produit pas de stratification thermique, la teneur en oxygène dissous est la même en surface et en profondeur, la décantation des éléments fins en suspension est faible et après quelques années n'entraîne pas de prélèvement sensible sur le transport solide par suspension. Il résulte de tout cela que la qualité des eaux n'est pas affectée par l'aménagement et que le littoral maritime n'est pas exposé à une régression par insuffisance des apports solides.

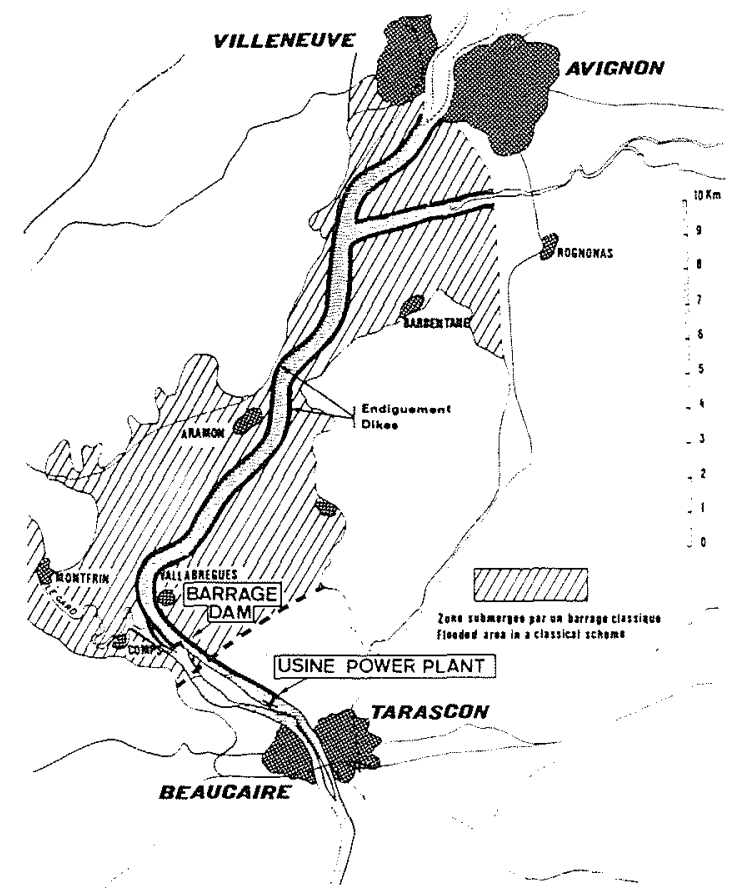

Figure 3 - Un exemple de réduction des emprises grace aux endiguements latéraux (Chute de Vallabrègues).

\begin{tabular}{|c|c|c|}
\hline $\begin{array}{l}\text { Aménagements } \\
\text { Development schemes }\end{array}$ & $\begin{array}{c}\text { Volume des retenues } \\
\text { volume of the } \\
\text { reservoirs } \\
10^{6} \mathrm{~m} .3\end{array}$ & $\begin{array}{c}\text { Durée de remplissage } \\
\text { Duration tor filing } \\
\text { déblt semi-perianent } \\
\text { (heures }\end{array}$ \\
\hline GENISSIAT & 53 & 47 \\
\hline SEYSSEL & 6 & 5 \\
\hline CHAUTAGNE & 7,8 & 6 \\
\hline BELLEY & 25,3 & 19 \\
\hline BREGNIER-CORDON & 13,6 & 10 \\
\hline SAULT-BRENAZ & 32,8 & 23 \\
\hline LOYETTES & 22,1 & 15 \\
\hline PIERRE-BENITE & 20 & 7 \\
\hline VAUGRIS & 22 & 8 \\
\hline PEAGE DE ROUSSILLON & 45 & 15 \\
\hline SAINT VALLIER & 35 & 12 \\
\hline BOURG LES VALENCE & 25 & 6 \\
\hline BEAUCHASTEL & 30 & 7 \\
\hline BAIX LOGIS NEUF & 40 & 9 \\
\hline MONTELIMAR & 45 & 10 \\
\hline DONZERE-MONORAGON & 20 & 4 \\
\hline CADEROUSSE & 37 & 8 \\
\hline AVIGNON & 34 & 7 \\
\hline VALLABREGUES & 70 & 13 \\
\hline
\end{tabular}

Figure 4 - Caractéristiques des retenues du Rhône.

La seconde conséquence est qu'une conduite sophistiquée de l'exploitation est nécessaire, d'autant que les marges de mancuvre par rapport aux crêtes d'endiguement sont très faibles (de l'ordre de $2 \mathrm{~m}$ ) et que des affluents à régime torrentiel très violent débouchent dans les aménagements. 


\section{Un projet à buts multiples}

Pour contraignant qu'il soit, le souci de réduire les emprises ne suffit pas, à lui seul, pour déterminer les aménagements. C'est la recherche du compromis le plus satisfaisant entre les objectifs poursuivis qui constitue le second fil directeur dans l'établissement des projets.

Il s'agit bien d'un compromis car, si l'on considère par exemple la production d'énergie et la navigation, on voit que, traités isolément, ces deux objectifs ne conduisent pas nécessairement au même projet.

En effet, les usines hydroélectriques de basse chute, équipées de groupes bulbes ou Kaplan, trouvent vraisemblablement leur optimum de rentabilité pour des chutes situées entre 15 et $25 \mathrm{~m}$. A l'inverse, les écluses de navigation, présentent le coût le plus faible, par mètre de chute, pour des dénivellations voisines de dix mètres, car il n'est pas nécessaire pour cette hauteur de réaliser des dispositifs de remplissage ou de vidange trop complexes et l'on amortit mieux l'ensemble des coûts fixes (garages de navigation, ouvrages de manœuvre des vannes et portes, cabine de commande, batardement, etc.) que dans le cas des très basses chutes.

De même, les canaux de navigation à grand gabarit se contentent d'une section mouillée de l'ordre de 200 à $250 \mathrm{~m}^{2}$. Au contraire, pour la production d'énergie, il faut, sur le Bas-Rhône, compte tenu des débits importants du fleuve, réaliser des canaux présentant une section de l'ordre de $1000 \mathrm{~m}^{2}$.

Enfin, la navigation entraîne diverses contraintes spécifiques telles que le rayon de courbure du chenal, le tirant d'air sous les ponts, la vitesse du courant, la limitation des ondes de disjonction.

En définitive, le découpage en chutes et le choix des cotes de retenues, sont déterminés par des considérations énergétiques car ce sont elles qui ont le poids économique le plus important. Il en est de même des canaux puisque les besoins énergétiques sont plus exigeants que ceux de la navigation. Par contre, les tracés sont définis de manière à respecter toutes les contraintes propres à la voie navigable (coupures de méandres dans les retenues, limitation des vitesses de courant dans les canaux, étude des tracés pour éviter les courants traversiers, etc.), et des dispositions spéciales sont prises vis-à-vis des ondes de disjonction.

Les études économiques ont montré très clairement que ce projet mixte était beaucoup plus intéressant qu'un projet couvrant un seul objectif en raison de l'importance des ouvrages communs. On a ainsi réalisé une voie navigable dont le coût au $\mathrm{km}$ est très inférieur à celui d'autres voies à grand gabarit, réalisées artificiellement, et la production d'énergie est d'un coût comparable à celui des ouvrages purement hydroélectriques réalisés par $\mathrm{EDF}$.

\section{La propagation des crues}

Ces aménagements posent de nombreux problèmes d'ordre hydraulique. La plupart sont localisés dans telle ou telle partie des ouvrages, par exemple :

- la dissipation d'énergie à l'aval des barrages,
- le tracé optimal des entrées et des sorties d'usines afin de réduire au minimum les pertes de charges, - le tracé des conduits de vidange et de remplissage des écluses,

- le dimensionnement correct des seuils déversants... etc.

Mais la question la plus importante et la plus générale est celle de la propagation des crues $[1,2]$. En effet, il subsiste des tronçons importants du Rhône qui ne sont pas endigués et où il est indispensable de ne pas aggraver les crues.

Or, la création de retenues à niveau peu variable tout au long d'une rivière, a normalement, pour effet, d'accélérer la propagation des crues et de réduire leur écrêtement naturel et, par conséquent, d'exhausser leur niveau dans les zones aval.

Pour résoudre ce problème, la CNR a d'abord dû créer un très important réseau de mesures hydrologiques qui permet, en particulier, de connaitre avec précision : d'une part, les hydrogrammes de crues dans toutes les sections caractéristiques, et d'autre part, les zones inondées. Puis, à partir de ces données, des modèles mathématiques en écoulement transitoire ont été établis pour reconstituer très fidèlement les phénomènes naturels, et déterminer les écoulements dans le Rhône aménagé en fonction de divers projets et diverses consignes d'exploitation.

Il a été ainsi possible de trouver une solution parfaitement satisfaisante puisque, non seulement il n'y a pas aggravation des crues mais au contraire, une large amélioration.

Les méthodes employées sont de trois ordres :

- conserver dans certains secteurs clefs, des zones inondables, mais faire en sorte que ces zones soient cependant inondées pour des débits supérieurs à ceux qui sont observés dans la nature; de cette manière, on dispose lors du débordement, d'un volume de stockage plus important qu'à l'état naturel. (C'est le cas, par exemple, de la plaine de Caderousse qui joue un rôle protecteur important pour la ville d'Avignon);

- réaliser dans certaines zones sensibles, des dragages importants qui abaissent les niveaux de crues suffisamment pour compenser les effets négatifs de l'aménagement. Ces dragages, qui sont effectués généralement en tête de retenue, ont en outre, l'intérêt d'abaisser les niveaux pour les débits moyens et donc d'augmenter la production de l'usine d'amont. Ils trouvent ainsi le plus souvent, une rentabilité propre sur le simple plan énergétique ;

- fermer partiellement ou totalement les usines hydroélectriques à un moment judicieusement choisi de la crue afin d'augmenter le débit transitant dans le tronçon de fleuve court-circuité par le canal de dérivation, et profiter ainsi de la possibilité de stockage offerte par le lit du Rhône en raison du prélèvement opéré auparavant dans le canal. Ce schéma d'exploitation est particulièrement important sur le Haut-Rhône où l'écrêtement des crues joue un rôle capital pour l'agglomération lyonnaise. (Fig. 5). 


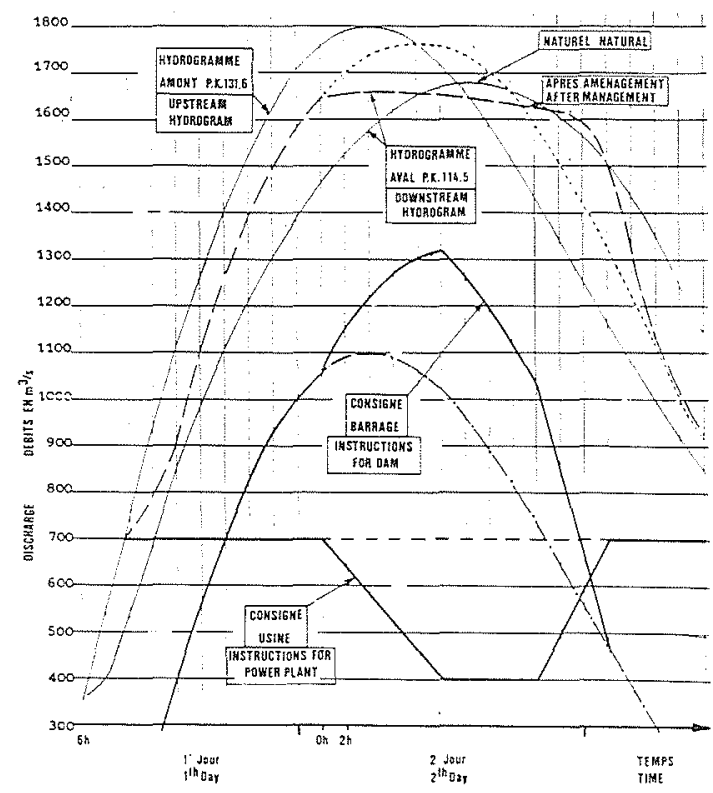

Figure 5 - Exemple de consigne d'exploitation en crue décennale (chute de Belley).

\section{Les nappes phréatiques [3]}

Les travaux de canalisation du Rhône, en créant une série de retenues endiguées et des canaux de dérivation, ont eu pour effet, d'exhausser le niveau du fleuve très largement au-dessus des lignes d'eau moyennes et quelquefois même, à quelques dix ou quinze mètres au-dessus des terres riveraines. Inversement, les canaux de fuite et le court-circuitage du débit par les canaux, ont pour effet, d'abaisser les lignes d'eau.

Or, la nappe alluviale de la vallée du Rhône est directement influencée par le fleuve,

- soit qu'il joue un rôle prépondérant dans son alimentation,

- soit qu'il règle son niveau dans toute la plaine riveraine, s'il s'agit d'une nappe de versant.

Cette nappe est très importante car elle est largement utilisée par l'agriculture et par des captages destinés à la fourniture d'eau potable ou industrielle.

L'expérience a montré qu'il était facile de prévenir les exhaussements de nappe, mais plus délicat d'empêcher les abaissements, aussi, d'une manière très générale, les projets sont beaucoup plus en relief qu'en creux et les canaux de fuite sont beaucoup plus courts que les canaux d'amenée

$\mathrm{La}$ prévention des exhaussements est obtenue par des canaux de drainage disposés le long des retenues (Fig. 6). Ces contre-canaux ont un double rôle :

- assurer le réglage des nappes phréatiques en décomprimant la piézomètrie en provenance de la retenue. Pour cela, il suffit que le toit des alluvions graveleuses soit entaillé par le contre-canal sur une hauteur de deux

(Les numéros entre crochets [ ] renvoient à la bibliographie en fin d'article). mètres environ, ou bien, si la couche supérieure des limons est trop épaisse, il faut percer cette couche par des forages pénétrant en profondeur dans les alluvions perméables et débouchant dans le contre-canal ;

- assurer l'évacuation des eaux de ruissellement de la plaine riveraine et des petits ruisseaux affluents dont les rives sont trop basses pour qu'ils puissent se jeter dans les retenues.

La géométrie des contre-canaux est ainsi définie par la double condition de présenter, pour le débit de percolation, une ligne d'eau suffisamment basse pour que la nappe se cale dans la plaine à son niveau moyen avant aménagement, et de ne pas donner lieu à des débordements en cas d'orage décennal.

Les débits de percolation à travers les endiguements sont relativement élevés au moment de la mise en eau (de l'ordre de $200 \mathrm{l} / \mathrm{s}$ par mètre de charge et par kilomètre de rive). Ils décroissent ensuite rapidement en raison du colmatage de l'endiguement, de sorte qu'il est nécessaire, dans les premières années de service de l'aménagement, de soutenir la ligne d'eau dans les contrecanaux par des petits seuils en enrochements. On peut ainsi ajuster empiriquement les nappes à leur meilleur niveau.

Le problème est plus complexe en milieu urbain. Il s'agit alors de régler les nappes pour éviter l'inondation des caves ou l'humidification des immeubles par remontée capillaire à partir de leurs fondations. Il faut assurer à la fois le drainage et l'évacuation des égouts qui sont le plus souvent unitaires et se jettent dans le Rhône par de multiples émissaires. Il faut obstruer tous ces émissaires et remanier complètement le système d'assainissement en créant de grands collecteurs

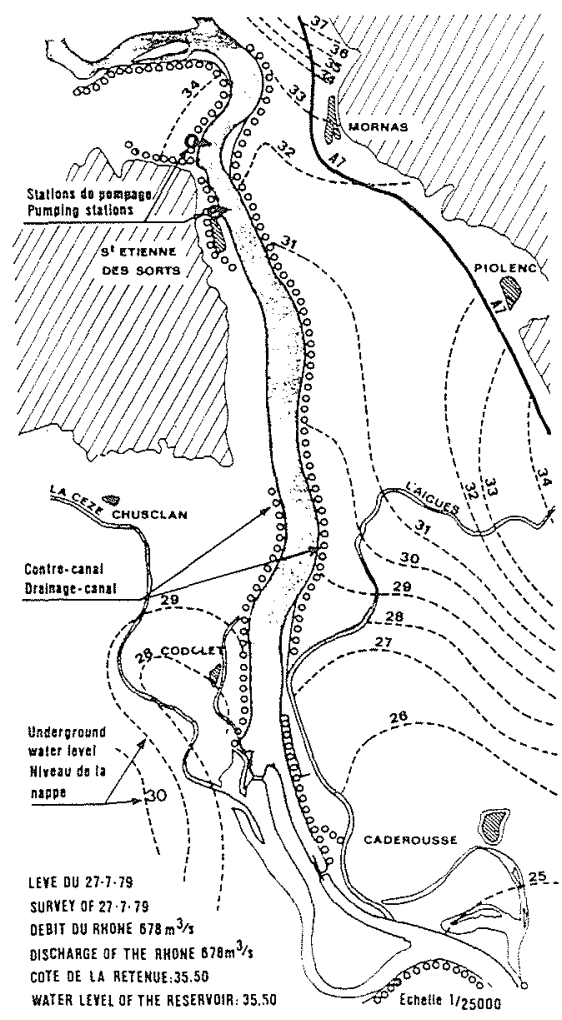

Figure 6 - Un exemple de contrôle de la nappe phréatique par les contre-canaux (chute de Cadérousse). 
riverains qui débouchent le plus souvent sur une station de pompage. La détermination des débits de drainage, qui est essentielle pour le calibrage des collecteurs et de la station de pompage, est rendue dans ce cas particulièrement difficile, car en milieu urbain, les rives sont souvent hétérogènes et constituées par des remblais divers pouvant donner lieu localement à de grosses arrivées d'eau. Ainsi, il arrive que la recherche de l'optimum économique conduise à réduire les arrivées d'eau de drainage par des tronçons de parois moulées disposées entre le collecteur et le Rhône dans les sections où la perméabilité est très élevée.

Ces dispositifs ont été réalisés par la Compagnie pour de nombreuses agglomérations riveraines du Rhône (Lyon, Vienne, Saint-Vallier, Tain l'Hermitage, Avignon notamment). Ils ont en outre l'avantage de maintenir les villes hors d'eau en période de crue tant que les débits de débordement ne sont pas atteints, alors qu'auparavant les sous-sols, caves et parkings souterrains étaient fréquemment inondés.

A l'inverse, comme on l'a indiqué ci-dessus, les cas d'abaissement de nappes sont plus difficiles à traiter. Ils se présentent en bordure des canaux de fuite et en bordure du Rhône court-circuité. Parfois mème, ils traversent le Rhône ou un canal de dérivation et se manifestent sur l'autre rive par l'effet de couches très perméables situées en profondeur.

On remédie à ces inconvénients en créant des réseaux d'irrigation et en approfondissant les puits de captage. Dans un cas exceptionnel, celui du canal de fuite de la chute de Donzère-Mondragon (le premier ouvrage du Bas-Rhône), la $\mathrm{CNR}$ a procédé à une réalimentation de la nappe, mais ce procédé est coûteux car il nécessite un renouvellement très fréquent des puits de réalisation en raison de leur colmatage. Il n'a donc pas été renouvelé et les ouvrages ultérieurs ont été conçus pour ne donner lieu qu'à des abaissements limités ne nécessitant pas de tels travaux.

\section{Conclusion}

La Compagnie Nationale đu Rhône en aménageant le Rhône, a tenté de résoudre le dilemme posé à tout aménageur fluvial : comment tirer le meilleur parti possible d'un fleuve au profit de l'économie de la nation, sans pour autant compromettre l'économie régionale en inondant la vallée par un haut barrage?

Cet objectif requiert des dispositions relativement complexes notamment en ce qui concerne la propagation des crues et la conservation des nappes phréatiques. L'aménagement du Rhône montre qu'il est possible de trouver des solutions efficaces au problème ainsi posé.

Ces solutions sont plus onéreuses, en ce qui concerne la production d'énergie, que la méthode plus classique du haut barrage, mais elles sont amplement justifiées si l'on considère la valeur des terres épargnées tant pour l'agriculture que pour l'urbanisme et l'industrie.

L'aménagement du Rhône est remarquable par la continuité de sa réalisation qui fut poursuivie sans relâche quoiqu'avec des rythmes divers, sur une longue période où cependant les données économiques et techniques ont extraordinairement varié. C'est sans doute la multiplicité des objectifs poursuivis qui a assuré cette continuité, malgré les fluctuations de la conjoncture. Mais c'est aussi, le souci permanent de la CNR de tirer le parti maximal des innovations technologiques et des enseignements de l'exploitation, qui a permis d'adapter de mieux en mieux, les projets aux objectifs.

\section{Références bibliographiques}

[1] SAVEY P. - Les effets de l'aménagement du Bas-Rhône sur l'écoulement des crues en régime permanent $S H F, X^{e}$ Journées de l'hydraulique 1968.

[2] MATHIAN J, - Incidence de l'aménagement du lit du Bas-Rhône sur la propagation des crues $S H F, X^{e}$ Journées de l'hydraulique 1968 .

[3] SAVEY P. et PONNELLE R. - Les effets de l'aménagement du Rhone sur la nappe phréatique, exemples de comportements singuliers SHF XIII Journées de l'hydraulique 1974.

\begin{tabular}{|c|c|}
\hline $\begin{array}{r}\text { Integrated development } \\
\text { A. General design and effect on }\end{array}$ & $\begin{array}{l}\text { ract } \\
\text { f a large river: the Rhone } \\
\text { loods and subsurface water layers }\end{array}$ \\
\hline $\begin{array}{l}\text { Responsibility for the development of the } 500 \mathrm{~km} \text { of the } \\
\text { French part of the Rhone is in the hands of the National } \\
\text { Rhône Corporation (CNR). } \\
\text { The operation is virtually completed to-day as } 16 \text { of the } 19 \\
\text { locations to be harnessed are already in service. } \\
\text { This is a multi-purpose development (hydro-electric gene- } \\
\text { ration, navigation by wide vessels, agricultural development, } \\
\text { protection against floods, port facilities, etc.). } \\
\text { The Rhône has promising potential with a semi-permanent } \\
\text { flow of } 1460 \mathrm{~m}^{3} / \mathrm{s} \text {., conveying an average } 50 \text { billions } \mathrm{m}^{3} \\
\text { per year. It produced } 16.9 \mathrm{TWh} \text { in } 1981 \text {. The navigable section } \\
\text { built as of that date is } 350 \mathrm{~km} \text { long and can handle the passage } \\
\text { of } 4000 \text { to } 5000 \text { ton convoys. } \\
\text { Given the fact that the valley is populated, the project has } \\
\text { been designed to occupy as little space as possible and to } \\
\text { reduce the absorption of land to the strict minimum. Conse- } \\
\text { quently, it includes very long lateral dykes and apart from } \\
\text { Genissiat upstream, head } 70 \mathrm{~m} \text {, is divided into a continuous } \\
\text { series of low heads. }\end{array}$ & $\begin{array}{l}\text { As a result, reservoirs are low by comparison with the } \\
\text { river's flow. This has a very positive effect on the quality of } \\
\text { the water and the transit of solid matter, but necessitates very } \\
\text { sophisticated operation to secure correct propagation of flood- } \\
\text { waters. } \\
\text { It is particularly important to prevent acceleration and } \\
\text { overtlowing of tloodwaters, albeit the floodable surfaces have } \\
\text { been significantly reduced by dykes and diversion canals. } \\
\text { This type of project also implies very tight control of } \\
\text { subsurface water layers, whether on farmable sites or in the } \\
\text { urban environment. The report shows the methods used to } \\
\text { master the phenomenon in particular using very large-scale } \\
\text { drainage devices. } \\
\text { The continuity of the work, which was spread over thirty } \\
\text { years, and the fact that the CNR is both designer and ope- } \\
\text { rator, have made it possible tor this development to benefit } \\
\text { at all times trom the signiticant technological progress which } \\
\text { has occurred in recent decades. }\end{array}$ \\
\hline
\end{tabular}

